Check for updates

Cite this: J. Mater. Chem. B, 2020 8, 7941

\section{Continuous production of uniform chitosan beads as hemostatic dressings by a facile flow injection method}

\author{
Boxuan Li, (D) $\dagger^{\mathrm{ab}}$ Juan Wang, (D) $\dagger^{\mathrm{bc}}$ Qin Gui ${ }^{\mathrm{b}}$ and Hu Yang (D) *bde
}

In this work, we developed a facile flow injection method to fabricate chitosan beads of uniform size in a continuous manner and assessed their properties as hemostatic dressings. Chitosan was dissolved into the ionic liquid 1-ethyl-3-methylimidazolium acetate (EMIM AC) to form a solution, and then the chitosan/EMIM Ac solution was flow injected into ethanol to form beads of uniform size. The formed chitosan beads were obtained following the exchange of ethanol solvent with water and freeze-drying. The overall process is ecofriendly and scalable without acid/alkali treatment or the use of cross-linking agents. The morphology, swelling, and cytotoxicity of the chitosan beads were characterized. The blood coagulation and whole blood clotting kinetics of the chitosan beads were also studied. The chitosan beads have a significantly high swelling capacity. They can absorb 30 times as much water and 40 times as much $\mathrm{PBS} / 0.9 \% \mathrm{NaCl}$ solution as their dry mass. Cytotoxicity was not observed on $\mathrm{NIH} 3 \mathrm{~T} 3$ fibroblast cells after $24 \mathrm{~h}$ - or $48 \mathrm{~h}$-incubation, when the concentration of the chitosan beads was $1.2 \mathrm{mg} \mathrm{mL}^{-1} \mathrm{or}$ lower. Pig whole blood quickly lost its flowability in 10 min after the blood $(1 \mathrm{~mL})$ was incubated with $1 \mathrm{mg}$ of chitosan beads. The whole blood clotting kinetics results showed that the chitosan beads efficiently caused the pig whole blood to clot with the absorbance of red blood cells (RBCs) dramatically reduced in $20 \mathrm{~min}$ and further reduced in 1 hour. The chitosan beads were shown to be biocompatible with excellent hemostatic properties. This work can apply a simple method to prepare chitosan beads for trauma hemostasis and broaden chitosan processing such as continuous manufacturing and 3D printing.
Received 10th June 2020, Accepted 14th July 2020

DOI: $10.1039 / d 0 t b 01462 a$

rsc.li/materials-b dissolving chitosan in acids and precipitating in alkalis. Many small molecules/macromolecules are involved in cross-linking. The process is tedious, and the acid and alkali residuals or cross-linkers may affect the biological properties of chitosan materials.

The preparation of chitosan materials by adjusting the chitosan hydrogen-bonded network itself in plain water is an eco-friendly way for its biomedical applications, which needs critical safety demand. Ionic liquids (ILs) are environmentally benign solvents as they have features such as non-volatility, thermal and chemical stability, and ease of recycling. ${ }^{17-20}$ Many water-miscible ionic liquids can dissolve nature-based polysaccharides. It opens an opportunity to use them as media for breaking and rebuilding the hydrogen-bonded network of chitosan in plain water. ${ }^{21,22}$ Recently, we developed an eco-friendly method to prepare plain water-based chitosan solution. ${ }^{23}$ In the method, we first solubilize chitosan in an ionic liquid, freeze the chitosan/IL system, and then subject it to extensive solvent change with water to remove the IL. The method provides a new approach to fabricate chitosan products. A drug-loaded chitosan film has been prepared with the plain-water based chitosan solution for improved drug delivery. ${ }^{24}$ In this work, we went on to develop a 
method to fabricate chitosan beads by integrating continuous flow injection into the process. In particular, chitosan/IL solution is flow injected into ethanol to form beads and remove IL, which is then washed with water and freeze-dried. The overall process is ecofriendly and potentially allows for large-scale continuous production. The morphology, swelling, cytotoxicity, blood coagulation, and whole blood clotting kinetics of the resulting chitosan bead were studied for its performance assessment as a potential hemostatic dressing. The chitosan products prepared by this method do not require acid/alkali treatment or the use of crosslinking agents, more appealing for biomedical applications.

\section{Materials and methods}

\section{Materials}

High molecular weight (HMW) chitosan (310 000-375 $000 \mathrm{Da})$, ionic liquid 1-ethyl-3-methylimidazolium acetate (EMIM Ac, $\geq 95.0 \%$ ) and WST-1 assay were purchased from SigmaAldrich. Ethanol, phosphate-buffered saline (PBS, $1 \times, \mathrm{pH}$ 7.4), sodium chloride ( $\mathrm{NaCl})$, and calcium chloride $\left(\mathrm{CaCl}_{2}\right)$ were purchased from Thermo Fisher Scientific. Water was ultrapurified by deionization and filtration before use. Pig whole blood (collected following the United States Department of Agriculture (USDA) guidelines with 3.8\% anticoagulant sodium citrate solution at $1: 10$ dilution) was purchased from Pel Freez Biologicals.

\section{Preparation of chitosan beads}

Chitosan (HMW) was dissolved into ionic liquid EMIM Ac at $\sim 115{ }^{\circ} \mathrm{C}$ to prepare a solution at a concentration of $20 \mathrm{mg} \mathrm{mL}^{-1}$. The chitosan/EMIM Ac solution was then injected into ethanol through a syringe with a $0.33 \mathrm{~mm}$ inner-diameter needle at room temperature. The injection speed was set at $10 \mathrm{~mL} \mathrm{~h}^{-1}$ controlled using a syringe pump (KD Scientific KDS 270 , USA). The resulting beads were kept in ethanol overnight and washed with ethanol three times to remove ionic liquid completely. Then the chitosan beads were washed with water three times to allow solvent exchange between ethanol and water in $30 \mathrm{~min}$. Finally, the hydrated chitosan beads were freeze-dried to obtain anhydrous chitosan beads. The sizes of the chitosan beads were measured by using the ImageJ software. ${ }^{25}$

\section{Scanning electron microscope (SEM)}

SEM images of chitosan beads were taken under a scanning electron microscope (Hitachi FE-SEM Su-70 and S-3400N, Japan) after being sputter-coated with gold-platinum for $90 \mathrm{~s}$.

\section{Swelling}

Lyophilized chitosan beads were pre-weighted as $W_{0}$ and then immersed and incubated at $37{ }^{\circ} \mathrm{C}$ in $1 \mathrm{~mL}$ of water, PBS (pH 7.4 ), or $0.9 \% \mathrm{NaCl}$ aqueous solution, respectively. At predetermined time points of $0.25,0.5,1,2,4,6$, and $24 \mathrm{~h}$, the hydrated chitosan beads were weighed as $W_{t}$. The swelling ratio (\%) is calculated as $\left(W_{t}-W_{0}\right) / W_{0} \times 100$.

\section{Cytotoxicity}

The cytotoxicity of chitosan beads was evaluated in NIH3T3 mouse embryo fibroblast cells by WST-1 assay as previously reported. ${ }^{26}$ Briefly, NIH3T3 cells were seeded in 24-well plates at a density of $1 \times 10^{5}$ cells per well. After cell attachment, chitosan beads were added to each well at a density of $0.4 \mathrm{mg} \mathrm{mL}^{-1}$, $0.8 \mathrm{mg} \mathrm{mL} \mathrm{m}^{-1}$, and $1.2 \mathrm{mg} \mathrm{mL}^{-1}$, respectively. After $24 \mathrm{~h}$ and $48 \mathrm{~h}$ incubation, the cell viability was tested on WST-1 assay using a microplate spectrophotometer.

\section{Blood coagulation tests}

Chitosan beads ( $1 \mathrm{mg}, 2 \mathrm{mg}$, and $5 \mathrm{mg}$ ) were added to $1 \mathrm{~mL}$ of pig whole blood as-purchased with an anticoagulant in a $37{ }^{\circ} \mathrm{C}$ water bath. Time was recorded right after the addition of $50 \mu \mathrm{L}$ of $0.1 \mathrm{M} \mathrm{CaCl}_{2}$ aqueous solution to the mixture. The tube was tilted every $30 \mathrm{~s}$ to observe if the blood was gelatinized. The clotting time was recorded when the blood completely lost flowability. ${ }^{27}$

\section{Whole blood clotting kinetics}

Clotting of $1 \mathrm{~mL}$ of pig whole blood was initiated by adding $50 \mu \mathrm{L}$ of $0.1 \mathrm{M} \mathrm{CaCl}_{2}$ aqueous solution. The initiated blood $(100 \mu \mathrm{L})$ was immediately added to a tube containing $1 \mathrm{mg}$, $2 \mathrm{mg}$, or $5 \mathrm{mg}$ of chitosan beads maintained at $37^{\circ} \mathrm{C}$. At each time point of $5,20,35$, and $50 \mathrm{~min}, 3 \mathrm{~mL}$ of water was added to each tube for $5 \mathrm{~min}$ to lyse red blood cells (RBCs), which were not trapped within the blood clot. The diluted hemoglobin released from the lysed RBCs was added to a 96 well plate ( $200 \mu \mathrm{L}$ per well) in triplicates for each sample and time point. The absorbance at $540 \mathrm{~nm}$ was recorded using a microplate reader. ${ }^{28}$

Statistical analysis. The data were reported as means \pm standard deviation (SD). Student's $t$-test was conducted for statistical analysis. $P$ values less than 0.05 were considered statistically significant.

\section{Results and discussion}

The flow injection process for the fabrication of chitosan beads is shown in Fig. 1. A dissolution process is the first step, in which chitosan (HMW) is solubilized in ionic liquid EMIM Ac at $115{ }^{\circ} \mathrm{C}$. Flow injection is subsequently carried out as the chitosan/EMIM Ac solution is dropwise injected to ethanol to form spherical chitosan beads. The resulting chitosan beads are collected, washed with ethanol, and then engaged in the solvent exchange process in water. Finally, freeze-drying is followed to dry chitosan beads.

The flow injection system is simple to set up and enables the bead fabrication process to be carried out easily. It is composed of a syringe pump and a receiver filled with ethanol, which is positioned on a magnetic stirrer (Fig. 2a). Continuous production of transparent pale yellowish chitosan beads with a diameter of $2.5 \pm 0.2 \mathrm{~mm}$ is enabled as chitosan/EMIM Ac solution is dispensed into ethanol at a controlled rate (Fig. 2b). The hydrogen-bonded network of chitosan macromolecular chains is disrupted by ionic liquid EMIM Ac through the dissolution 


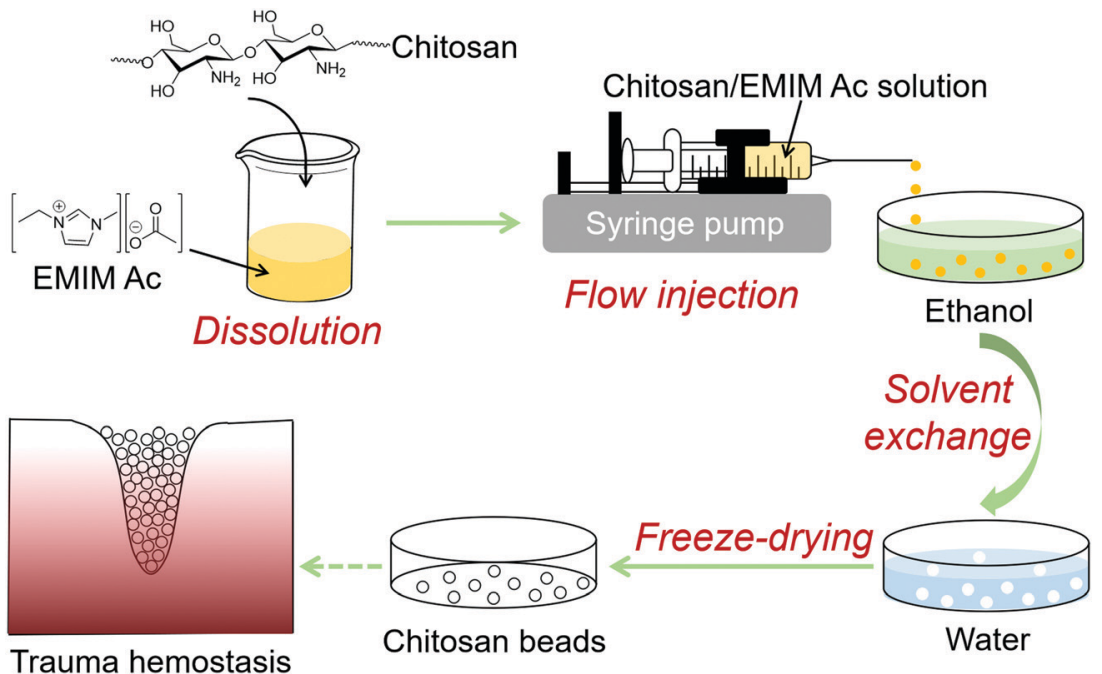

Fig. 1 Schematic illustration of the fabrication of chitosan beads through a flow injection process.
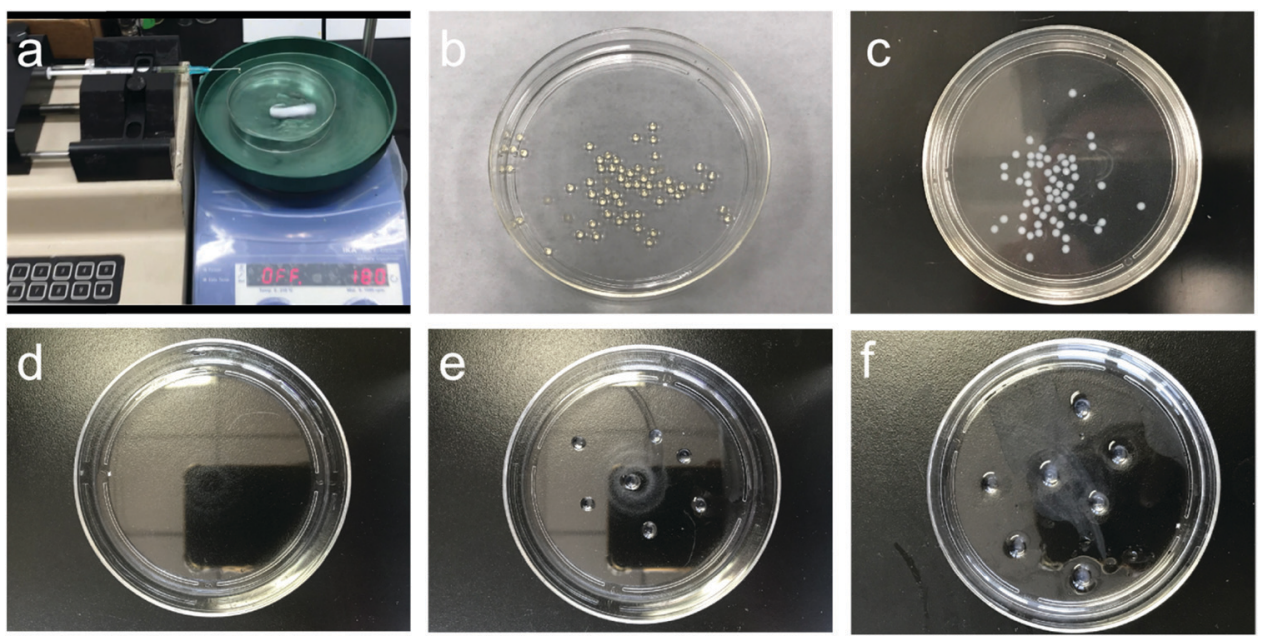

Fig. 2 The flow injection system setup and monitoring of chitosan beads throughout the process. (a) Flow injection experimental setup. (b) Chitosan beads produced upon the injection of chitosan/EMIM Ac to ethanol. (c) Chitosan beads after ethanol wash. The appearance of chitosan beads following ethanol wash for various lengths of time, i.e., $10 \mathrm{~min}$ (d), $30 \mathrm{~min}$ (e), and overnight (f) and subsequent 30 min solvent exchange with water.

process. ${ }^{22}$ When chitosan/EMIM Ac is added to a nonsolvent like ethanol, chitosan/EMIM Ac drop immediately collapses and stabilizes in spherical shape. As the ionic liquid EMIM Ac was washed out with ethanol and the hydrogen-bonded network was rebuilt, the chitosan beads gradually turned white (Fig. 2c). The chitosan beads retrieved from the ionic liquid maintained relatively stable shape and size $(2.6 \pm 0.1 \mathrm{~mm})$ in ethanol.

The subsequent shape-maintenance of chitosan beads in water depends strongly on its hydrogen-bonded network rebuilding through the ethanol-based washing process. It is especially important to control the duration of the ethanol washing before the solvent exchange with water. If we directly injected chitosan/EMIM Ac into the water without ethanol processing, we could only get a chitosan water solution. This is because the amine groups of chitosan would be gradually protonated during the solvent exchange process from ionic liquid EMIM Ac to water. ${ }^{23}$ It reduces the cross-linking effect from the hydrogen-bonding interactions of chitosan through electrostatic repulsion. A water-based gel-like solution of chitosan would form, which was also observed in our previous study. ${ }^{23}$ When the ethanol washing step was less than $10 \mathrm{~min}$, the ionic liquid largely residues in chitosan beads, and there is no shaped chitosan material left after removing the washing water for $30 \mathrm{~min}$ solvent exchange with stirring (Fig. 2d). Extending ethanol washing to $30 \mathrm{~min}$ helped preserve chitosan beads $(4.2 \pm 0.2 \mathrm{~mm})$ (Fig. 2e). To further eliminate the effect of ionic liquid residues, we prolonged the ethanol washing overnight, during which ethanol was refreshed three times. As a result, the chitosan beads were maximally preserved in water despite that their size increased to $4.6 \pm 0.5 \mathrm{~mm}$ due to swelling (Fig. 2f). ${ }^{24}$ In this process, the hydrogen-bonded network of 

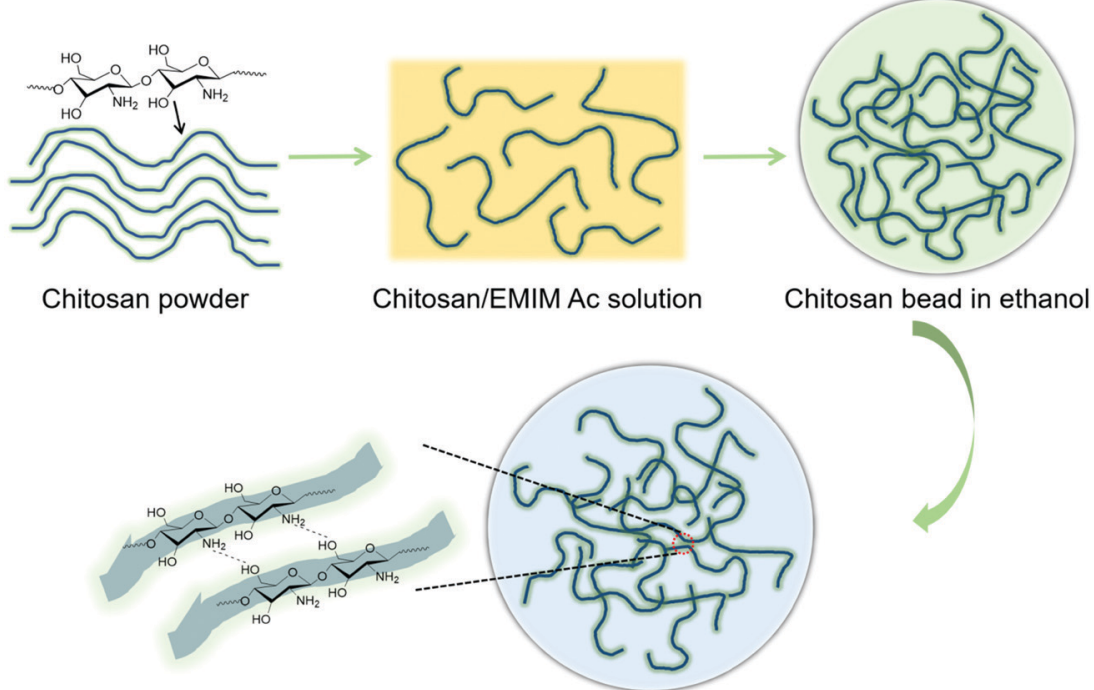

Chitosan bead in water

Fig. 3 Proposed chitosan macromolecular chain formation from chitosan powder, chitosan/EMIM Ac solution and formed chitosan bead in ethanol and water.

chitosan was broken by the dissolution of EMIM Ac and successfully rebuilt as a cross-linking effect for the chitosan beads in ethanol. This cross-linking effect has largely remained after the subsequent solvent exchange process with water. A micron-sized bulky structure-chitosan bead was formed in water by the cross-linking effect of chitosan hydrogen-bonding interactions itself (Fig. 3).

The freeze-dried chitosan beads appear as solid white particles (Fig. 4a) and have an average size of $3.5 \pm 0.4 \mathrm{~mm}$ (Fig. 4b). The surface of the chitosan bead shrunk and formed a compact wrinkled microstructure (Fig. 4c), which is typically seen in freeze-dried materials. ${ }^{26}$ The internal microstructure appears to be porous (Fig. 4d). The swelling behaviors of dehydrated chitosan beads were studied in water, $\mathrm{PBS}$, and $0.9 \% \mathrm{NaCl}$ solution equilibrated at $37^{\circ} \mathrm{C}$. As shown in Fig. 5 , chitosan beads
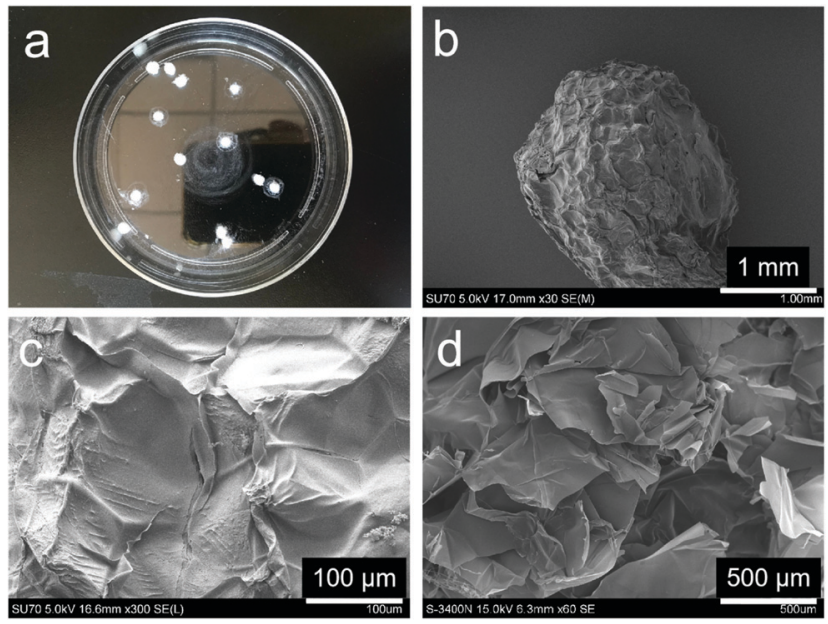

Fig. 4 Photograph and SEM images of freeze-dried chitosan beads. (a) Photograph of chitosan beads. SEM images of a whole chitosan bead (b), the surface morphology (c), and the internal structure (d) of chitosan bead.

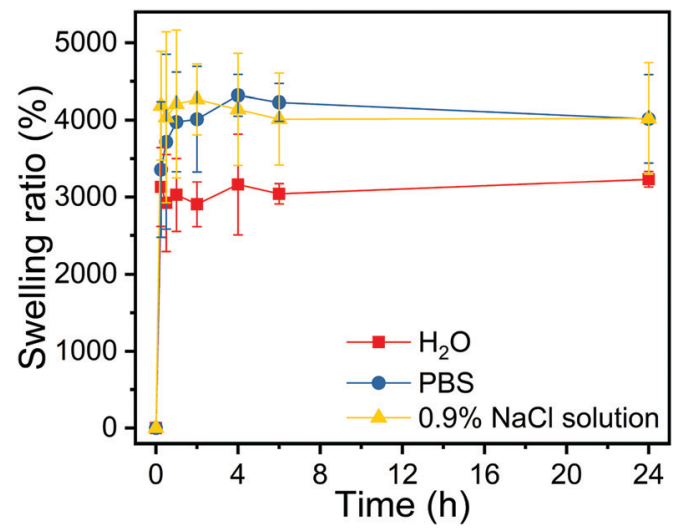

Fig. 5 Swelling behavior of dried chitosan beads in water, PBS $(\mathrm{pH} 7.4)$ and $0.9 \% \mathrm{NaCl}$ solution at $37{ }^{\circ} \mathrm{C}$.

reached swelling equilibrium within 60 min regardless of the medium type. Chitosan beads absorbed 30 times as much water as their weight. More impressively, they absorbed nearly 40 times

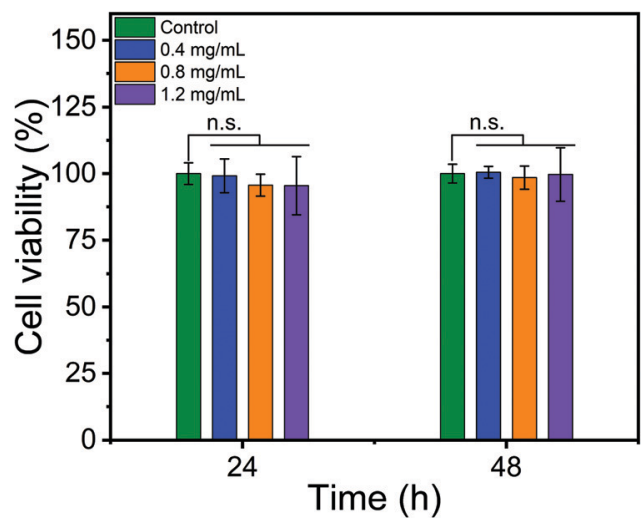

Fig. 6 Cytotoxicity of chitosan beads to NIH3T3 cells. n.s. indicates not significant. 

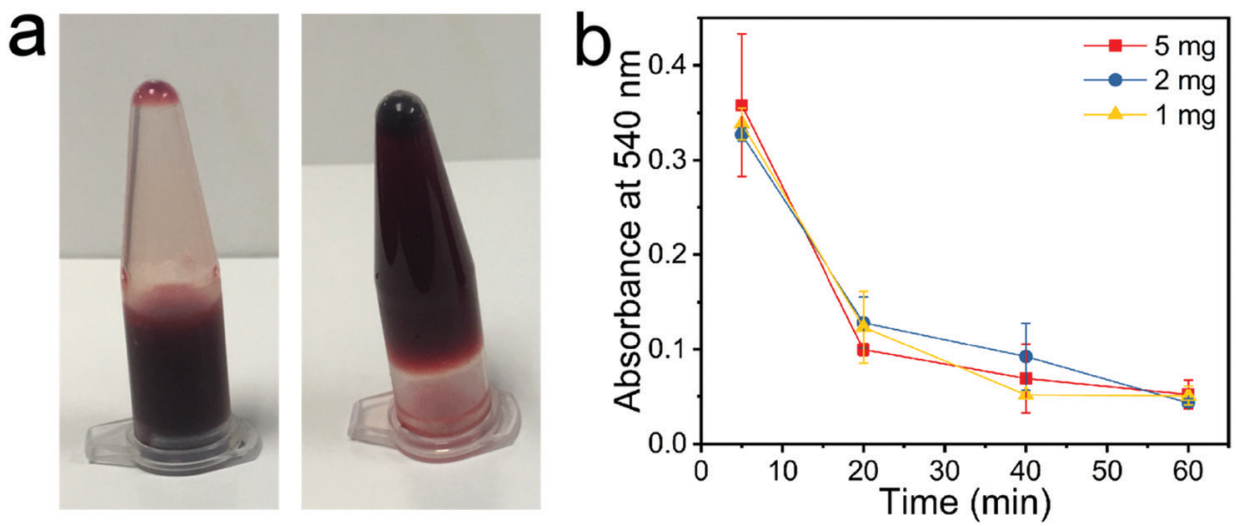

Fig. 7 Hemostatic properties of chitosan beads. (a) Pig whole blood $(1 \mathrm{~mL})$ coagulated upon the addition of $1 \mathrm{mg}$ of chitosan beads. Left panel: Untreated pig whole blood; right panel, the blood stopped flowing after 10 min treatment with chitosan beads. (b) Pig whole blood clotting kinetics induced by various amounts of chitosan beads.

as much PBS or $0.9 \% \mathrm{NaCl}$ solution as their weight. The significantly high swelling capacity of the chitosan beads is attributed to their high hydrophilicity and porous microstructures. The chitosan beads were shown to have much higher swelling in PBS and $0.9 \% \mathrm{NaCl}$ solution due to their higher affinity to salt solutions. ${ }^{29-31}$

The chitosan beads were shown to possess excellent cytocompatibility. NIH3T3 fibroblast cells did not experience viability reduction following $24 \mathrm{~h}$ or $48 \mathrm{~h}$ incubation with chitosan beads within the concentration range of 0.4 and $1.2 \mathrm{mg} \mathrm{mL}^{-1}$ (Fig. 6). Given the utility of chitosan-based materials for wound healing and hemostasis, ${ }^{22,27,32,33}$ we tested the hemostatic properties of the chitosan beads prepared by us. Pig whole blood quickly lost its flowability in 10 min upon the addition of $1 \mathrm{mg}$ chitosan beads to $1 \mathrm{~mL}$ of the blood (Fig. 7a). Nonetheless, higher amounts of chitosan beads ( $2 \mathrm{or} 5 \mathrm{mg}$ ) did not seem to accelerate blood coagulation. The whole blood clotting kinetics induced by chitosan beads was further studied. The absorbance is indicative of the number of red blood cells (RBCs) not trapped within the clot. Therefore, the absorbance of RBCs is used to monitor clotting kinetics. Chitosan beads efficiently caused the pig whole blood to clot, as evidenced by the dramatic reduction in RBC absorbance in 20 min and a further reduction in 1 hour (Fig. 7b). Similar whole blood clotting kinetics was observed among the three treatment groups, consistent with blood coagulation test results.

Our preliminary assessment supports the potential use of chitosan beads as hemostatic dressings prepared using this simple ecofriendly flow injection method. Based on this method, it is possible to prepare chitosan beads of different sizes by adjusting injection parameters, e.g., the needle gauge, flow rate, stirring speed, and rheological properties of chitosan/IL systems. Chitosan beads can be used to deliver drugs. For instance, water-soluble drugs can be loaded on chitosan beads through surface adsorption, and water-insoluble or sparingly soluble drugs can be blended into chitosan during the dissolution by ionic liquids. ${ }^{34,35}$ The ionic liquid can be recycled and reused for reducing the overall manufacturing cost. Taken together, this simple continuous flow injection method provides a new opportunity to fabricate scalable chitosanbased biomedical materials and drug-loaded formulations.

\section{Conclusions}

In this work, we developed a simple flow injection method that enables the continuous production of chitosan beads of uniform size. Chitosan is first dissolved in ionic liquid EMIM Ac and injected into ethanol at a controlled rate using an infusion pump for bead forming, followed by solvent exchange with water and freeze-drying. The resulting chitosan beads have uniform size and porous microstructures. Chitosan beads possess high swelling capability and good biocompatibility. They are capable of efficiently inducing blood coagulation and clotting and have the potential use as hemostatic dressings for trauma hemostasis. This flow injection method provides a new way to fabricate chitosan products in a continuous manufacturing fashion.

\section{Conflicts of interest}

The authors declare no competing financial interest.

\section{Acknowledgements}

This work was supported, in part, by the Fundamental Research Funds for the Central Universities (Grant No. 3332019100 and 2019PT320028) (BL).

\section{References}

1 M. Rinaudo, Chitin and chitosan: properties and applications, Prog. Polym. Sci., 2006, 31, 603-632.

2 M. Dash, F. Chiellini, R. M. Ottenbrite and E. Chiellini, Chitosan-A versatile semi-synthetic polymer in biomedical applications, Prog. Polym. Sci., 2011, 36, 981-1014.

3 M. Rodriguez-Vazquez, B. Vega-Ruiz, R. Ramos-Zuniga, D. Alexander Saldana-Koppel and L. Fernando Quinones-Olvera, 
Chitosan and its potential use as a scaffold for tissue engineering in regenerative medicine, BioMed Res. Int., 2015, 821279.

4 K. Azuma, S. Ifuku, T. Osaki, Y. Okamoto and S. Minami, Preparation and biomedical applications of chitin and chitosan nanofibers, J. Biomed. Nanotechnol., 2014, 10, 2891-2920.

5 G. Cardenas, P. Anaya, C. von Plessing, C. Rojas and J. Sepulveda, Chitosan composite films. Biomedical applications, J. Mater. Sci.: Mater. Med., 2008, 19, 2397-2405.

6 J.-D. Mathias, N. Tessier-Doyen and P. Michaud, Development of a chitosan-based biofoam: application to the processing of a porous ceramic material, Int. J. Mol. Sci., 2011, 12, 1175-1186.

7 P. S. Barber, S. P. Kelley, C. S. Griggs, S. Wallace and R. D. Rogers, Surface modification of ionic liquid-spun chitin fibers for the extraction of uranium from seawater: seeking the strength of chitin and the chemical functionality of chitosan, Green Chem., 2014, 16, 1828-1836.

8 K. Zhu, J. Duan, J. Guo, S. Wu, A. Lu and L. Zhang, Highstrength films consisted of oriented chitosan nanofibers for guiding cell growth, Biomacromolecules, 2017, 18, 3904-3912.

9 Y. Yang, X. Wang, F. Yang, H. Shen and D. Wu, A universal soaking strategy to convert composite hydrogels into extremely tough and rapidly recoverable double-network hydrogels, Adv. Mater., 2016, 28, 7178-7184.

10 D. Zhu, X. Jin, X. Leng, H. Wang, J. Bao, W. Liu, K. Yao and C. Song, Local gene delivery via endovascular stents coated with dodecylated chitosan-plasmid DNA nanoparticles, Int. J. Nanomed., 2010, 5, 1095-1102.

11 I. Wedmore, J. G. McManus, A. E. Pusateri and J. B. Holcomb, A special report on the chitosan-based hemostatic dressing: experience in current combat operations, J. Trauma Acute Care Surg., 2006, 60, 655-658.

$12 \mathrm{X}$. Shu and K. J. Zhu, A novel approach to prepare tripolyphosphate/chitosan complex beads for controlled release drug delivery, Int. J. Pharm., 2000, 201, 51-58.

13 F. C. Wu, R. L. Tseng and R. S. Juang, Comparative adsorption of metal and dye on flake- and bead-types of chitosans prepared from fishery wastes, J. Hazard. Mater., 2000, 73, 63-75.

14 A. K. Anal, D. Bhopatkar, S. Tokura, H. Tamura and W. F. Stevens, Chitosan-alginate multilayer beads for gastric passage and controlled intestinal release of protein, Drug Dev. Ind. Pharm., 2003, 29, 713-724.

15 F. L. Mi, H. W. Sung and S. S. Shyu, Drug release from chitosan-alginate complex beads reinforced by a naturally occurring cross-linking agent, Carbohydr. Polym., 2002, 48, 61-72.

16 M. Li, Z. Wang and B. Li, Adsorption behaviour of congo red by cellulose/chitosan hydrogel beads regenerated from ionic liquid, Desalin. Water Treat., 2016, 57, 16970-16980.

17 R. D. Rogers and K. R. Seddon, Ionic liquids-solvents of the future?, Science, 2003, 302, 792-793.

18 K. R. Seddon, Ionic liquids: a taste of the future, Nat. Mater., 2003, 2, 363-365.

19 T. Welton, Room-temperature ionic liquids. Solvents for synthesis and catalysis, Chem. Rev., 1999, 99, 2071-2084.
20 C. D. Hubbard, P. Illner and R. van Eldik, Understanding chemical reaction mechanisms in ionic liquids: successes and challenges, Chem. Soc. Rev., 2011, 40, 272-290.

21 R. P. Swatloski, S. K. Spear, J. D. Holbrey and R. D. Rogers, Dissolution of cellose with ionic liquids, J. Am. Chem. Soc., 2002, 124, 4974-4975.

22 S. S. Silva, J. F. Mano and R. L. Reis, Ionic liquids in the processing and chemical modification of chitin and chitosan for biomedical applications, Green Chem., 2017, 19, 1208-1220.

23 B. Li, J. Wang, M. E. Moustafa and H. Yang, Ecofriendly method to dissolve chitosan in plain water, ACS Biomater. Sci. Eng., 2019, 5, 6355-6360.

24 B. Li, J. Wang, Q. Gui and H. Yang, Drug-loaded chitosan film prepared via facile solution casting and air-drying of plain water-based chitosan solution for ocular drug delivery, Bioact. Mater., 2020, 5, 577-583.

25 C. A. Schneider, W. S. Rasband and K. W. Eliceiri, NIH image to Image J: 25 years of image analysis, Nat. Methods, 2012, 9, 671-675.

26 J. Wang, H. He, R. C. Cooper and H. Yang, In situ-forming polyamidoamine dendrimer hydrogels with tunable properties prepared via aza-Michael addition reaction, ACS Appl. Mater. Interfaces, 2017, 9, 10494-10503.

27 X. Sun, Z. Tang, M. Pan, Z. Wang, H. Yang and H. Liu, Chitosan/kaolin composite porous microspheres with high hemostatic efficacy, Carbohydr. Polym., 2017, 177, 135-143.

28 C. Liu, X. Liu, C. Liu, N. Wang, H. Chen, W. Yao, G. Sun, Q. Song and W. Qiao, A highly efficient, in situ wet-adhesive dextran derivative sponge for rapid hemostasis, Biomaterials, 2019, 205, 23-37.

29 A. Salam, J. J. Pawlak, R. A. Venditti and K. El-tahlawy, Synthesis and characterization of starch citrate-chitosan foam with superior water and saline absorbance properties, Biomacromolecules, 2010, 11, 1453-1459.

30 A. Salam, R. A. Venditti, J. J. Pawlak and K. El-Tahlawy, Crosslinked hemicellulose citrate-chitosan aerogel foams, Carbohydr. Polym., 2011, 84, 1221-1229.

31 A. Ayoub, R. A. Venditti, J. J. Pawlak, A. Salam and M. A. Hubbe, Novel hemicellulose-chitosan biosorbent for water desalination and heavy metal removal, ACS Sustainable Chem. Eng., 2013, 1, 1102-1109.

32 T. Dai, M. Tanaka, Y. Y. Huang and M. R. Hamblin, Chitosan preparations for wounds and burns: antimicrobial and wound-healing effects, Expert Rev. Anti-Infect. Ther., 2011, 9, 857-879.

33 R. A. Muzzarelli, Chitins and chitosans for the repair of wounded skin, nerve, cartilage and bone, Carbohydr. Polym., 2009, 76, 167-182.

34 M. Moniruzzaman and M. Goto, Ionic liquids: future solvents and reagents for pharmaceuticals., J. Chem. Eng. Jpn., 2011, 44, 370-381.

35 N. Adawiyah, M. Moniruzzaman, S. Hawatulailaa and M. Goto, Ionic liquids as a potential tool for drug delivery systems, MedChemComm, 2016, 7, 1881-1897. 